\section{Should Clinical Practitioners, as Part of Institutional or Accreditation Standards, Be Required to Document Their Rationale When Choosing to Not Adhere to Widely Accepted Clinical Practice Guidelines?}

\section{THE "PRO" SIDE}

Clinician documentation in the health record is an essential tool in patient care. It establishes accountability and responsibility, and it facilitates transparency for decisionmaking. ${ }^{1}$ Patient assessments, care plans, interventions, and other activities are recorded by virtually all multidisciplinary team members working in inpatient settings, including nurses, physiotherapists, dietitians, social workers, and physicians. Pharmacists document medication histories and reconciliation, patient counselling interviews, therapeutic drug monitoring, and recommendations for resolution of drug-related problems. In fact, pharmacists may be trained to cite the primary literature or a clinical practice guideline (CPG) when writing a recommendation in the patient chart. ${ }^{2}$ Is it unreasonable, then, that clinicians be expected to record when they are choosing therapeutic pathways incongruent with best available evidence?

CPGs, defined as "systematically developed statements to assist practitioner and patient decisions about appropriate health care for specific clinical circumstances", have been devised to augment several aspects of patient care. ${ }^{3}$ One such feature of quality patient care is patient safety. Adherence to CPGs, through use of protocols and checklists, for example, has been shown to reduce adverse patient outcomes through improved standardization and communication. ${ }^{4}$ Minimizing patient harm is also fundamental to the composition of institutional accreditation standards, which are increasingly the chief vehicle for safety initiatives within these settings. ${ }^{5}$ Recognizing that miscommunication is implicated in an overwhelming majority of medical errors, the Joint Commission International Accreditation Standards for Hospitals outline that the care provided to each patient is to be planned and written in the patient's record. ${ }^{6,7}$

Another consideration favouring documentation of nonadherence with CPGs is the principle that patients with the same health problems and care needs have a right to receive the same quality of care throughout an organization, despite the variations that exist within and among areas of practice. ${ }^{4,7}$ To achieve "one level of quality of care", services provided to comparable populations in multiple departments or settings may be guided by policies and procedures that result in their uniform delivery. ${ }^{7}$ CPGs can influence equity of patient care through enhanced consistency in therapeutic decision-making, and organizations may deter discrepancy by obligating clinicians to justify departures from known best practice with documented rationale.

It must also be acknowledged that sharing of information across disciplines and between care settings is essential for effective collaboration in achieving desired patient outcomes. ${ }^{7}$ Insufficient data transmission contributes to stress on both patients and staff, generates additional workload, and prolongs patient visits. ${ }^{89}$ Record-keeping systems facilitate information flow that supports the continuity, quality, and safety of patient care. Shared understanding of a patient's care history facilitates sound intra- and inter-disciplinary communication and decision-making about future care. ${ }^{10}$ Accreditation standards prescribe that institutions design and execute processes to provide continuity of patient care services through organization and coordination among personnel. Endorsement of documented CPGs is one way to integrate coordinated care among multidisciplinary care providers within and across health care settings.

CPGs are intended to influence health professionals' actions, and thereby, ultimately, the patient's outcome, but compliance is notoriously lacking. ${ }^{11}$ Opponents might protest that adherence to CPGs should not be mandatory, that they are not, in fact, "one size fits all" solutions, and that patient care decisions need to remain clinically flexible. According to this rationale, enforcement of such therapeutic protocols, and additional documentation thereof, serve to disrespect physician autonomy and specialized clinical expertise. Irrespective of these perceived patient interests, deviations from CPGs compromise improvements in care among populations. ${ }^{12} \mathrm{CPG}$ are developed according to best available evidence, and many medical professionals have difficulty assimilating the rapidly evolving scientific evidence into their own practices. ${ }^{13} \mathrm{CPG}$ can provide a much-needed interface between research and practice that may contribute to more effective and efficient patient care. Of additional interest to physicians, evidence shows that standardization of care not only improves patient outcomes but also has a positive effect on malpractice litigation. ${ }^{14}$

Another anticipated challenge to realizing this new care norm is time. In addition to the requirement that care be dispensed according to a guideline, it may be considered an unreasonable burden to overwhelm busy physicians with expectations to document the rationale behind specific decisionmaking. However, clinical decision support technology through electronic health records is an evolving approach to improving 
guideline-based care and is easing such documentation through automation. ${ }^{15,16}$ Such computerization would also facilitate the evaluation of an institution's consistent implementation and use of CPGs in the clinical care pathways of patients, as required by accrediting agencies. ${ }^{7}$

The process to develop CPG-driven patient care protocols and associated clinician documentation of adherence must be inclusive and multidisciplinary and may need to be adapted to the local practice setting. Input from physicians into the review and distribution of CPGs is essential for fostering "buy-in", and procedures should be in place to notify and train end-user practitioners whenever such policies are to be implemented. Continuous quality improvement of any patient care initiative depends on a structured and well-defined data-driven process that is constantly monitored and improved; requiring clinical practitioners to document their rationale when they choose not to adhere to widely accepted CPGs is one such important mechanism.

\section{References}

1. Jorgenson D. Fulfilling our professional duty through documentation. Can Pharm J. 2008;141(2):76-7.

2. Farrell B, Kennie N, Dolovich L. Demonstrating value, documenting care: lessons learned about writing comprehensive patient medication assessments in the IMPACT project: Part I: Getting started with documenting medication assessments. Can Pharm J. 2008;141(3):114-9.

3. Clinical practice guidelines we can trust [consensus report]. Washington (DC): Institute of Medicine; 2011 Mar 23 [cited 2013 Apr 28]. Available from: www.iom.edu/Reports/2011/Clinical-Practice-Guidelines-We-CanTrust.aspx

4. American College of Obstetricians and Gynecologists Committee on Patient Safety and Quality Improvement. ACOG Committee Opinion No. 526: Standardization of practice to improve outcomes. Obstet Gynecol. 2012; 119(5):1081-2.

5. Warburton RN. Perspective on safety. Accreditation and regulation: can they help improve patient safety? In: Web MerM: morbidity and mortality rounds on the web. Rockville (MD): US Department of Health and Human Services, Agency for Healthcare Research and Quality; 2009 Apr [cited 2013 Apr 20]. Available from: www.webmm.ahrq.gov/perspective.aspx?perspectiveID=74

6. Dingley C, Daugherty K, Derieg MK, Persing R. Improving patient safety through provider communication strategy enhancements. Rockville (MD): US Department of Health and Human Services, Agency for Healthcare Research and Quality; [cited 2013 Apr 24]. Available from: www.ahrq.gov/downloads/pub/advances2/vol3/advances-dingley_14.pdf

7. Joint Commission International accreditation standards for hospitals. 4th ed. Oakbrook Terrace (IL): Joint Commission International; 2010.

8. Van Walraven C, Taljaard M, Bell CM, Etchells E, Zarnke KB, Stiell IG, et al. Information exchange among physicians caring for the same patient in the community. CMAJ. 2008;179(10):1013-8.

9. Mack Remen V, Grimsmo A. Closing information gaps with shared electronic patient summaries- how much will it matter? Int J Med Inform. 2011;80(11):775-81.

10. Keenan GM, Yakel E, Tschannen D. Chapter 49: Documentation and the nurse care planning process. In: Hughes RG, editor. Patient safety and quality: an evidence-based handbook for nurses. AHRQ Publ No. 08-0043. Rockville (MD): US Department of Health and Human Services, Agency for Healthcare Research and Quality; 2008.

11. Cabana MD, Rand CS, Powe NR, Wu AW, Wilson MH, Abboud PA, et al. Why don't physicians follow clinical practice guidelines? A framework for improvement. JAMA. 1999;282(15):1458-65.

12. Hoeksema LJ, Bazzy-Assad A, Lomotan EA, Edmonds DE, RamirezGarnica G, Shiffman RN, et al. Accuracy of a computerized clinical decisionsupport system for asthma assessment and management. J Am Med Inform Assoc. 2011;18(3):243-50.
13. Gerhardt WE, Schoetker PJ, Donovan EF, Kotogal UR, Muething SE, et al. Putting evidence-based clinical practice guidelines in to practice. An academic pediatric center's experience. Jt Comm J Qual Patient Saf. 2007; $33(4): 226-35$

14. Kirkpatrick DH, Burkman RT. Does standardization of care through clinical guidelines improve outcomes and reduce medical liability? Obstet Gynecol. 2010;116(5):1022-26.

15. Lyng KM. From clinical practice guidelines, to clinical guidance in practice-impacts of computerization. Int J Med Inform 2012 Oct 29. DOI 10.1016/j.ijmedinf.2012.10.004

16. Isern D, Moreno A. Computer-based execution of clinical guidelines: a review. Int J Med Inform. 2008;77(12):787-808.

Wessam El Kassem, BScPharm, MBA

Asma Abdulrahman, BScPharm

Ahmed Raghab, BScPharm

PharmD students

Kerry Wilbur, BSc(Pharm), ACPR, PharmD

Associate Professor and Director, Doctor of Pharmacy Program

College of Pharmacy

Qatar University

Doha, Qatar

Competing interests: None declared.

\section{THE "CON" SIDE}

Clinical practice guidelines (CPGs) are tools created to aid clinical decision-making and provide clinicians with evidencebased recommendations regarding patient care. Guidelines are meant to be developed on the basis of the most rigorous scientific research and should use expert opinion and consensus only when high-quality evidence is unavailable. ${ }^{1}$ When these standards are adhered to, uptake of guideline recommendations may lead to standardized care and optimized drug utilization. However, lack of generalizability to complex patients, misinterpretation of evidence, and high risk of actual or potential conflicts of interest commonly occur and preclude guidelines from being fully adopted into clinical practice. ${ }^{2}$

Clinical practitioners, as part of institutional or accreditation standards, should not be required to document rationale when choosing to not adhere to widely accepted CPGs. Simply put, there is insufficient evidence that guideline adherence positively affects patient outcomes. Before promoting adherence to guidelines as part of institutional and accreditation standards, clinicians, administrators, and patients must be convinced of their value. Most studies designed to assess this question have reported suboptimal guideline adherence and inconclusive results. ${ }^{3}$ These low adherence rates may reflect poor generalizability and/or a lack of clinician confidence in the validity of guideline recommendations.

In addition, implementing such a policy would threaten advancement of patient-centred care and perhaps cause regression of progress achieved to date. Individualization of drug therapy regimens would decrease, as clinicians would undoubtedly fear accreditation-related or institution-based repercussions for not adhering to accepted guidelines. This would bias 
clinicians to act in the best interest of the institution, rather than the best interest of the patient. Furthermore, guidelines are usually disease-focused and do not offer clear recommendations for patients with multiple disease states who are taking multiple medications. ${ }^{2}$ Unfortunately, these are the types of patients most commonly encountered in hospitals and health care institutions, and clinicians must maintain the freedom to perform and act upon individual patient assessment to avoid resorting to guideline-induced, "cookie cutter" medicine.

The influence of bias in guideline creation, from both internal and external parties such as financial contributors, must also be considered. Pharmaceutical companies desiring to promote product placement may directly or indirectly fund guideline authors. Additionally, disease-based organizations and advocacy groups may receive funding from these companies and may consequently have actual or potential conflicts of interest when endorsing guidelines. ${ }^{4}$ If the suggested documentation becomes mandatory, we fear that biased recommendations will be adopted more frequently, potentially putting patients at risk of receiving suboptimal therapy. These points are even more relevant for recommendations that are based on lower-quality evidence, such as observational studies and expert opinion. Shockingly, it has been shown that widely endorsed guidelines contain recommendations based on expert opinion or standard care on average $48 \%$ of the time. ${ }^{5}$

Aside from lack of evidence and the potential for harm, adopting a requirement to document the rationale for any deviation from widely accepted CPGs would pose logistical concerns that would be very difficult, if not impossible, to overcome. For example, it is unknown how "widely accepted" would be defined and how institutions and accreditation bodies would choose the specific guidelines to be endorsed. Also, clinicians would need to be educated and trained on each guideline and would have to be aware of the exact circumstances that would require documentation. Without the development of protocols and preprinted orders, this is not a realistic expectation for clinical practitioners. Finally, monitoring and evaluation plans would be essential for uptake and continued evaluation. Are health authorities ready to accept the burden of these extra costs with no clear evidence of improved patient outcomes?

Some may believe that enforcing this documentation strategy (through institutional and accreditation policies) will benefit patients and standardize care. They may argue that it will make prescribers more accountable for their actions. Although we support achieving competency standards and communication through documentation, we believe that this strategy will make prescribers accountable to the institution and to guideline creators, rather than the patient. By doing so, patients will lose autonomy in their care and be at risk of suboptimal therapy.

Instead, we support critical evaluation of CPGs and the incorporation of only high-quality, evidence-based recommendations into clinical care. Although clinical practitioners should be encouraged to document their rationale for decision-making, accreditation bodies should avoid using guideline adherence as a surrogate marker for competency and should instead maintain a focus on patient outcomes as markers for assessment. International bodies should continue to work with guideline creators to ensure that only high-quality documents with minimal potential for bias are disseminated. Once these principles are achieved, guidelines may be better suited for full endorsement and adoption.

In summary, clinical practitioners, as part of institutional or accreditation standards, should not be required to document their rationale when choosing to not adhere to widely accepted CPGs. Documentation should instead be focused on the rationale for decision-making, whether it is guidelines, primary literature, or other reasons. Additionally, the international community should advocate for rigorous evaluation mechanisms for CPGs and avoidance of adopting recommendations that are based on low-quality evidence. By doing so we hope that the best possible patient outcomes can be achieved through the use of evidence-based medicine and patient-centred care.

\section{References}

1. Carter A. Clinical practice guidelines. CMAJ. 1992;147(11):1649-50.

2. Shaneyfelt TM, Centor RM. Reassessment of clinical practice guidelines. Go gently into that good night. JAMA. 2009;301(8):868-9.

3. Cabana MD, Rand CS, Powe NR, Wu AW, Wilson MH, Abboud PC, et al. Why don't physicians follow clinical practice guidelines? A framework for improvement. JAMA. 1999;282(15):1458-65.

4. Conflicts of interest and development of clinical practice guidelines. In: Institute of Medicine, Committee on Conflict of Interest in Medical Research, Education, and Practice; Lo B, Field MJ, editors. Conflict of interest in medical research, education, and practice. Washington (DC): National Academies Press; 2009 [cited 2013 Apr 17]. Available from: www.ncbi.nlm.nih.gov/books/ NBK22928/

5. Tricoci P, Allen JM, Kramer JM, Califf RM, Smith SC. Scientific evidence underlying the ACC/AHA clinical practice guidelines. JAMA. 2009; 301(8):831-41.

\author{
Dina E Elgaily, BSC(Pharm) \\ Arwa O Sahal, BSc(Pharm) \\ Bassant A Orabi, BSc(Pharm) \\ Dina A lbrahim, BSc(Pharm) \\ PharmD students \\ Kyle J Wilby, PharmD \\ Assistant Professor, Clinical Pharmacy and Practice \\ College of Pharmacy \\ Qatar University \\ Doha, Qatar
}

Competing interests: None declared.

The Pro and Con articles for this issue's "Point Counterpoint" column were developed from a debate held in spring 2013 as part of the course "Pharmacy Research, Evaluation and Presentation Skills IV" in the Doctor of Pharmacy program at Qatar University. 